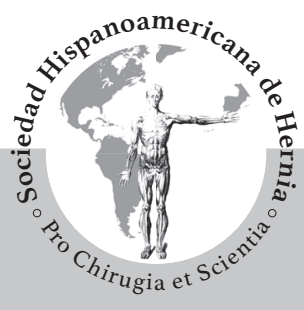

\title{
Estudio de relación entre el músculo psoas y el plexo lumbar retroperitoneal: implicaciones médico-quirúrgicas
}

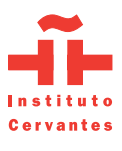
Anatomicall references between the psoas muscle
and the lumbar plexus in the retroperitoneal space-
Medicall and surgicall implications

\section{Alfredo Moreno-Egea ${ }^{1,2}$, Alfredo Moreno Latorre ${ }^{3}$}

1Jefe Clínica Hernia. Hospital Universitario La Vega. Murcia (España). ${ }^{2}$ Profesor de Anatomía Quirúrgica. Departamento de Anatomía Humana. Facultad de Medicina. Universidad Católica San Antonio (UCAM). Murcia (España). ${ }^{3}$ Residente de MFC. Servicio de Urgencias. Hospital Santa Lucía. Cartagena, Murcia (España)

Recibido: $12-11-2018$

Aceptado: 16-11-2018

Palabras clave:

Plexo lumbar, nervios, denervación, músculo psoas

\section{Resumen}

Introducción: El abordaje laparoscópico retroperitoneal para la exposición de la zona lateral lumbar puede causar lesiones del plexo lumbar. El objetivo de este estudio es proporcionar puntos de referencia anatómicos con el fin de prevenir lesiones neurales.

Material y método: Para estudiar la anatomía aplicada en relación con el plexo lumbar se utilizaron 10 cadáveres preservados con formaldehído en la Cátedra de Anatomía de la Facultad de Medicina de la Universidad Católica de Murcia. Se utilizó un colorante (azul de metileno) para valorar la disposición del plexo lumbar a través del músculo psoas.

Resultados: El plexo lumbar discurre por el músculo psoas y sus ramas terminales salen al espacio retroperitoneal en su borde lateral (iliohipogástrico e ilioinguinal) o lo perforan (genitofemoral en su cara anterosuperior, femorocutáneo posterolateralmente). Se describen referencias anatómicas neurales de origen y de salida al espacio retroperitoneal. La inyección de colorante en el interior del músculo psoas produce una diseminación global que afecta a todos los nervios del plexo lumbar. Las variaciones en la formación de los nervios, respecto de sus raíces de origen, son casi del $50 \%$.

Conclusión: El músculo psoas es una referencia quirúrgica básica en el abordaje retroperitoneal. Debe evitarse su borde superior y lateral. Los nervios GF (único o como dos ramas) y FCL discurren entre sus fascículos y deben localizarse antes de actuar sobre el MP. La gran variabilidad de formación en las raíces de los nervios terminales del plexo lumbar debe tenrese en cuenta al planear cualquier sección basada solo en el mapeo clínico. El bloqueo del compartimento del psoas es una posibilidad de anestesia regional de gran espectro neural.

*Autor para correspondencia: Alfredo Moreno Egea. Clínica Hernia. Hospital Universitario La Vega. C/ Dr. Román Alberca, s/n. 3008 Murcia (España) Correo electrónico: morenoegeaalfredo@gmail.com 
Key words:

Lumbar plexus, nerves, muscle

denervation, psoas muscle.

\begin{abstract}
Introduction: The retroperitoneal approach for exposing the lumbar lateral area can cause lesions of the lumbar plexus. The aim of this study is to provide anatomical landmarks in order to prevent neural lesions

Methods: To study the applied anatomy in relation to the lumbar plexus, 10 cadavers preserved with formaldehyde were used in the Anatomy Chair of the Faculty of Medicine of the Catholic University of Murcia. A dye (methylene blue) was used to assess the arrangement of the lumbar plexus through the psoas muscle.

Results: The lumbar plexus runs through the psoas muscle and its terminal branches exit into the retroperitoneal space at its lateral border (iliohypogastric and ilioinguinal) or perforate it (genitofemoral on its anterosuperior face, femorocutaneous posterolaterally). Neural anatomical references of origin and output to the retroperitoneal space are described. The injection of dye into the psoas muscle produces a global spread that affects all the nerves of the lumbar plexus. The variations in the formation of the nerves, with respect to their roots of origin, are almost $50 \%$.

Conclusion: The psoas muscle is a basic surgical reference in the retroperitoneal approach, and its upper and latera borders must be avoided. The genitofemoral nerve (single or as two branches) and femorocutaneous nerve run between their fascicles and must be located before acting on the psoas muscle. The great variability of formation in the roots of the terminal nerves of the lumbar plexus should be taken into account when planning any section based only on clinical mapping. Blockade of the psoas compartment is a possibility of regional anesthesia of a large neural spectrum.
\end{abstract}

\section{INTRODUCCIÓN}

El plexo lumbar se forma por las ramas anteriores de los tres primeros nervios lumbares y la mayor parte de los ramos anteriores de L4 y parte del duodécimo nervio intercostal. El primer trayecto neural (o de origen) discurre dentro del músculo psoas (MP) y no es valorable por el cirujano cuando aborda el espacio retroperitoneal en multitud de cirugías de diversas especialidades. El segundo trayecto cruzando el espacio retroperitoneal, lateral al MP, ya es visible y puede interpretarse por los especialistas en base a los conocimientos anatómicos disponibles ${ }^{1,2}$.

El plexo lumbar puede ser lesionado al colocar trocares en el abordaje laparoscópico retroperitoneal, al exponer la columna en una vía a través del MP o en cirugía urológica y ginecológica ${ }^{3-6}$. El principal problema de estos abordajes, además de la hemorragia parietal, es la posible denervación del flanco por lesión nerviosa. Esta complicación se presenta en el posoperatorio como un abultamiento o tumor abdominal lateral y/o una debilidad prolongada a la flexión de la cadera, y puede valorarse mediante electromiografía si muestra una actividad y reclutamiento reducido sobre los músculos laterales y psoas ${ }^{7-12}$

Por otro lado, los abordajes anterolaterales permiten un acceso directo al MP y se ha referido la posibilidad de utilizar esta vía para realizar bloqueos intraoperatorios. Este abordaje evitaría el bloqueo percutáneo, que presenta un considerable riesgo de hemorragia por la punción. La inyección directa en el MP durante una operación puede producir el bloqueo del compartimento del psoas y evitar la morbilidad asociada a la punción, complementando la analgesia posoperatoria ${ }^{13-15}$.

Para mejorar todos estos abordajes y procedimientos es necesario revisar los conocimientos neuroanatómicos lumbares, ya que no existen buenas descripciones anatómicas de la disposición del plexo lumbar en el espacio muscular del psoas, 10,16-21. El propósito de este estudio es aportar más datos anatómicos sobre el trayecto inicial del plexo lumbar y el compartimento plexal del psoas para aumentar la seguridad quirúrgica y prevenir posibles lesiones neurales.

\section{MATERIAL Y MÉTODO}

Para el estudio se han utilizado diez cadáveres preservados con formaldehído procedentes de la Cátedra de Anatomía de la Facul- tad de Medicina de la Universidad Católica de Murcia (UCAM). El estudio fue aprobado por el Comité de Ética del centro. La edad promedio de los cadáveres fue de 71 años, con un rango de 64-88 años, 7 hombres y 3 mujeres.

Se realizaron 20 disecciones completas del plexo lumbar, tanto de su trayecto retroperitoneal como intermuscular (simulando un abordaje anterior poserolateral), para identificar correctamente los nervios terminales: iliohipogástrico (Ih) e Ii, subcostal (espinal XII), femorocutáneo lateral (FCL), genitofemoral (GF), femoral (NF) y obturador (NO). También se disecaron en detalle los músculos psoas (MP), ilíaco (MI), cuadrado lumbar (MCL) y transverso del abdomen (MTA), sus respectivas aponeurosis y/o fascias (lámina anterior de la fascia toracolumbar y aponeurosis lumboilíaca) y las estructuras en relación con la espina ilíaca anterosuperior (EIAS).

Se inyectó un vial de azul de metileno bajo visión directa dentro del músculo psoas, separando el plano superior de inserción en los cuerpos vertebrales. Para la inyección del colorante se usó una aguja de calibre 18 sobre el primer cuerpo de inserciones musculares. No fue necesario profundizar en la inserción de la aguja de punción. El curso del azul de metileno, dentro de los dos planos del MP, fue visualizado directamente y observado abriendo el resto de inserciones sobre los cuerpos y apófisis costiformes vertebrales. Las preparaciones fueron registradas fotográficamente para su posterior análisis por un segundo observador. Los datos fueron recogidos de forma prospectiva y analizados después de forma descriptiva.

\section{RESULTADOS}

\section{Relación anatómica plexo lumbar-músculo psoas}

En nuestras disecciones encontramos el plexo lumbar formándose en su mitad posterior y saliendo al espacio retroperitoneal en su borde lateral en IH e Ii, o atravesándolo como el GF. El FCL puede salir lateral, en el borde del MI o perforarlo poserolateralmente. Las referencias anatómicas neurales respecto del MP se muestran en la figura 1 .

\section{Relación anatómica plexo lumbar-retroperitoneo}

Los nervios subcostal (XII espinal), IH e Ii se disponen de forma lateral al MP, y en oblicuo cruzando el MCL hasta alcanzar 


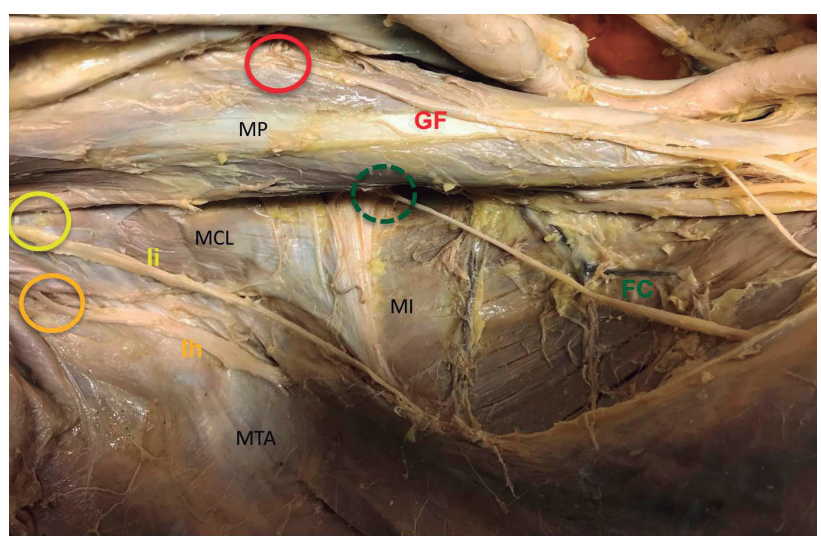

Figura 1. Referencias de salida del plexo lumbar al retroperitoneo en relación al músculo psoas. MCL: músculo cuadrado lumbar; MP: músculo psoas; MI: músculo ilíaco; MTA: músculo transverso abdominal; IH: nervio iliohipogástrico; Ii: nervio ilioinguinal; FC: nervio femorocutáneo; GF: nervio genitofemoral.

el MTA. Solo en un caso observamos división del nervio IH antes de penetrar el MTA; en el resto, siempre encontramos un tronco único en el espacio retroperitoneal. La referencia anatómica más destacable del subcostal es el ligamento arciforme lateral, borde inferomedial, y la última costilla; del IH, el vientre muscular más posterior del MTA; y del Ii, el punto de inserción del MTA sobre la cresta ilíaca. El nervio GF, único o en sus ramas, penetra el MP y discurre adherido a su fascia, sin divisiones hasta su tercio inferior, cerca del ligamento inguinal. Las referencias anatómicas neurales del espacio retroperitoneal se muestran en la tabla I y en la figura 2.

\section{Estudio del compartimento del psoas}

En las muestras inyectadas con el colorante de azul de metileno, el efecto de teñido es rastreado por el resto del espacio retroperitoneal sobre MP, MCL, MI y MTA. La valoración tras la extirpación completa del MP mostró un patrón de diseminación completo del colorante entre los dos planos de inserción medial del MP, dibujando el denominado espacio plexal. No detectamos fuga apreciable al resto de la cavidad, lo que confirma que la inyección dentro del MP en cadáveres produce un patrón de diseminación susceptible de afectar a todos los nervios terminales que componen el plexo lumbar, y podría utilizarse simulando un bloqueo completo (inguinal y de miembro inferior) del plexo lumbar (figs. 3 y 4 ).

\section{Estudio de formación del plexo lumbar}

Habitualmente se describe el plexo de la siguiente manera: los nervios IH e Ii proceden de L1; los nervios FCL y GF, de L2, y el NO-NF, de las raíces anteriores de L2-3-4. Esta disposición solo la encontramos en 5 cadáveres; en el resto (50\%), el origen fue diferente. La figura 5 muestra un caso de variación en el que el nervio IH nace de T12; el Ii, de L1 con el FCL como tronco común; del L2 nace el GF y de L3-4-5, el NF y NO. La figura 6 muestra otra variación donde el IH e Ii nacen de raíz diferente (T12 y L1, respectivamente) y el GC y FCL, también de raíz diferente (GC de L2 y FCL de L3). Las variaciones en la formación de los nervios, respecto de sus raíces de origen, son casi la norma, según nuestra experiencia anatómica.

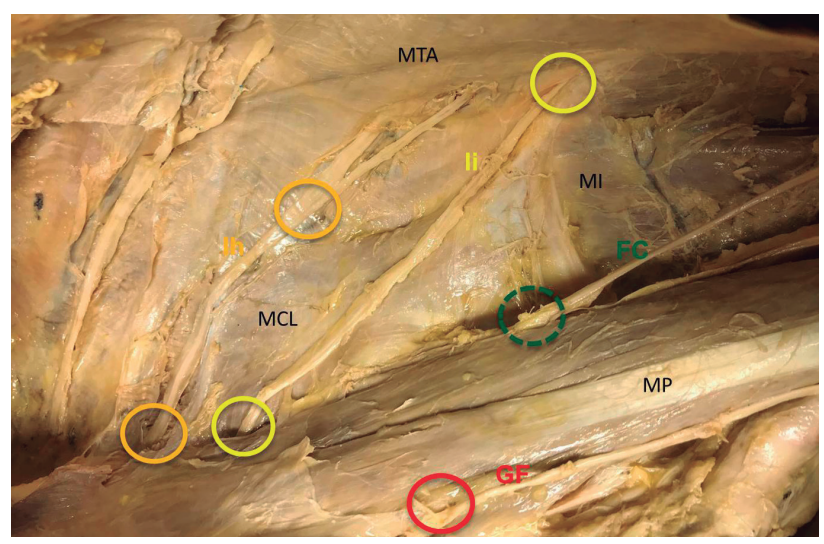

Figura 2. Referencias de salida del plexo lumbar al retroperitoneo y trayecto hasta su trayecto intermuscular en relación al músculo cuadrado lumbar. MCL: músculo cuadrado lumbar; MP: músculo psoas; MI: músculo iliaco; MTA: músculo transverso abdominal; IH: nervio iliohipogástrico; Ii: nervio ilioinguinal; FC: nervio femorocutáneo; GF: nervio genitofemoral.

\begin{tabular}{lll}
\multicolumn{2}{c}{ Tabla I. Referencias anatómicas del plexo lumbar en el espacio retroperitoneal } \\
\multicolumn{1}{c}{ Referencia } & \multicolumn{1}{c}{ De origen } & De salida \\
\hline Duodécimo intercostal & $\begin{array}{l}\text { Borde medial Lig. arciforme externo } \\
\text { Digitaciones D-MTA }\end{array}$ & Borde inferior de última costilla \\
Iliohipogástrico & Lateral al MP sobre borde muscular curvo del MCL & $\begin{array}{l}\text { 3-4 cm superior al MCL (Brouet) } \\
\text { Punto de transición aponeurosis - MTA }\end{array}$ \\
Ilioinguinal & mitad inferior del cuerpo muscular del CL & Cabestrillo muscular \\
Femoral cutáneo lateral & Vértice MP-MI & Borde óseo posterior de cresta ilíaca \\
Genitofemoral & Superficie anterior MP & Tercio lateral bajo ligamento inguinal \\
Femoral & Surco intermuscular MP-MI & Entre las dos espinas ilíacas \\
\hline
\end{tabular}

Lig.: ligamento; D: diafragma; MCL: músculo cuadrado lumbar; MP: músculo psoas; MTA: músculo transverso abdominal; MI: músculo ilíaco. 


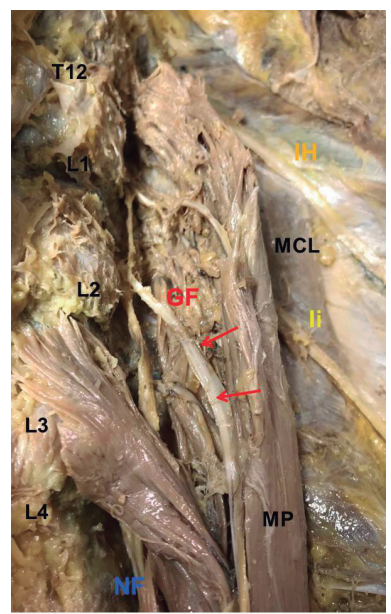

Figura 3. Espacio intermuscular del psoas al seccionar sus fascículos superiores: valoración del trayecto del nervio genitodemoral. Cuerpos de vértebras lumbares L1-L4; IH: nervio iliohipogástrico; Ii: nervio ilioinguinal; GF: nervio genitofemoral; NF: nervio femoral; MP: músculo psoas; MCL: cuadrado lumbar.

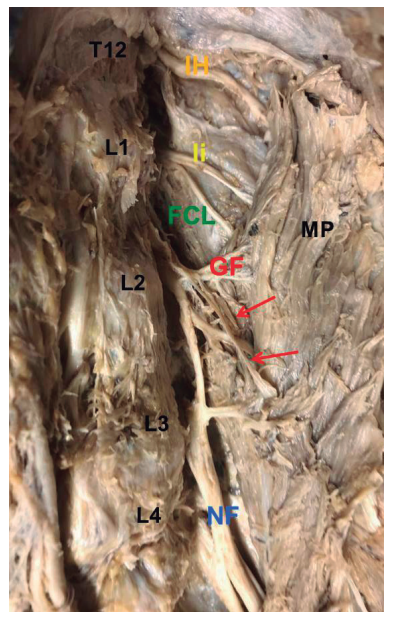

Figura 5. Variante de origen del plexo lumbar: IH de T12 e Ii y FCL de L2. IH: nervio iliohipogástrico; Ii: nervio ilioinguinal; FC: nervio femorocutáneo; GF: nervio genitofemoral; NF: nervio femoral; FCA: femorocutáneo anterior (rama del femoral); L1-4: cuerpos vertebrales lumbares; MP: músculo psoas; MCL: cuadrado lumbar; GF: nervio genitofemoral (sus ramas se indican con flechas rojas); FC: femorocutáneo lateral).

\section{DISCUSIÓN}

El abordaje laparoscópico ha supuesto una revolución en la práctica quirúrgica. Sin embargo, aunque se considera como un abordaje poco traumático y doloroso, la posibilidad de denervación de la pared posterolateral del abdomen por lesión nerviosa y formación de un tumor o pseudohernia debe tenerse en cuenta como complicación indeseable ${ }^{22}$. De igual forma, la sección que se realiza en el tratamiento del dolor inguinal crónico a nivel retroperitoneal (triple neurectomía por laparoscopia), deja la inervación de la pared poste-

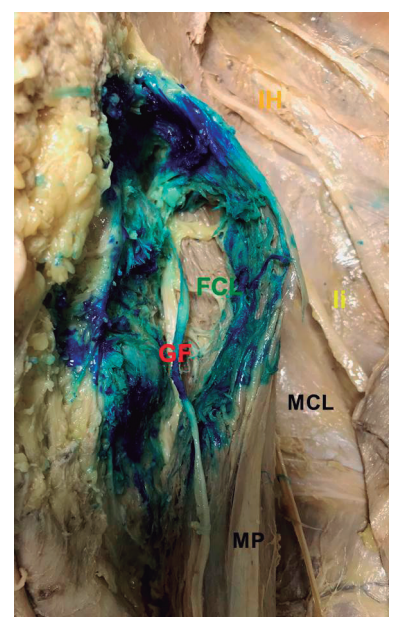

Figura 4. Tinción del espacio compartimental plexal: valoración de genitofemoral y femorocutáneo lateral como nervios más afectados. MCL: músculo cuadrado lumbar; MP: músculo psoas; IH: nervio iliohipogástrico; Ii: nervio ilioinguinal; FC: nervio femorocutáneo; GF: nervio genitofemoral.

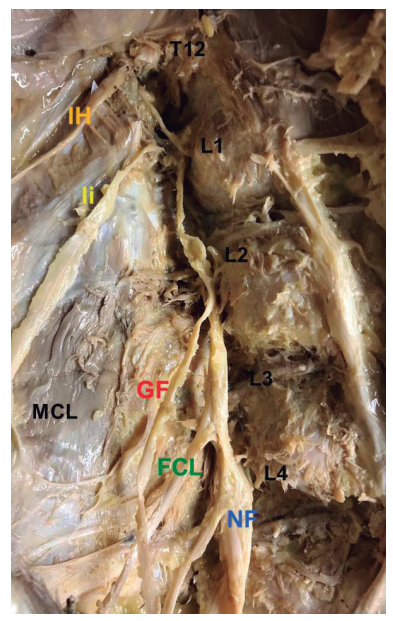

Figura 6. Variante de origen del plexo lumbar: IH de T12 e Ii y FCL de L2. IH: nervio iliohipogástrico; Ii: nervio ilioinguinal; FC: nervio femorocutáneo; GF: nervio genitofemoral; NF: nervio femoral; FCA: femorocutáneo anterior (rama del femoral); L1-4: cuerpos vertebrales lumbares; MP: músculo psoas; MCL: cuadrado lumbar; GF: nervio genitofemoral (sus ramas se indican con flechas rojas); FC: femorocutáneo lateral).

rolateral a cargo únicamente del nervio subcostal (12. lumbar), lo que también predispone a la posible aparición de una pseudohernia latera ${ }^{23}$. Otras técnicas y procedimientos (de columna, urológicos y ginecológicos) también pueden conllevar como efecto adverso la lesión del plexo lumbar ${ }^{10-20}$. Nuestro estudio muestra las referencias anatómicas de los nervios del plexo lumbar en el espacio retroperitoneal, con especial hincapié en la superficie del MP.

El tratamiento del dolor debe estar cimentado en un enfoque multidisciplinar y global a lo largo de todo el proceso médico-quirúrgico de un paciente. El bloqueo clásico del plexo lumbar se realiza de forma subcutánea bajo control de las referencias óseas externas sobre 


\begin{tabular}{llllll}
\multicolumn{6}{c}{ Tabla II. Variantes del nervio genitofemoral: estudio } \\
de la literatura
\end{tabular}

$\mathrm{N}$ : número de plexos lumbares analizados; Tipo 1: tronco único emergiendo del músculo psoas; Tipo 2: dos ramas independientes desde el espesor del músculo psoas; No: ausencia del nervio; L1-2: origen en las raíces lumbar 1 y 2 , resto del porcentaje como variantes)

la superficie del paciente. Esta técnica puede conllevar complicaciones importantes, como el hematoma retroperitoneal, lesión renal, punción subaracnoidea o propagación epidural ${ }^{24-27}$. Por ello, buscando otras alternativas, se propuso el bloqueo del compartimento del psoas, cuya base técnica es la ubicación anatómica de las raíces nerviosas lumbares dentro del MP (donde se forman los nervios GF, FCL, NF y NO). En este espacio, una sola inyección de agente anestésico puede bloquear todo el contenido neural que lo atraviesa y causar lo que se denomina un bloqueo tipo 3 en 1 (GF-NF-NO) ${ }^{28-30}$. Nosotros, como advertimos en este estudio, comprobamos que la difusión de un anestésico en el MP puede extenderse fácilmente a todos sus nervios, incluyendo el IH e Ii, según las necesidades de tratamiento del dolor, posibilidad que hemos reproducido en la sala de disección, siguiendo las experiencias de Green en $2011^{30}$.

Para realizar procedimientos seguros en el espacio retroperitoneal es necesario ampliar nuestros conocimientos en neuroanatomía del plexo lumbar ante la gran distorsión de conocimientos de los textos clásicos que se usan como enseñanza básica de residentes y especialistas ${ }^{30-34}$. Nuestro estudio verifica las frecuentes variantes de origen de los nervios terminales, lo que limita la extrapolación de conclusiones fiables ante un mapeo clínico de sus raíces respecto de sus dermatomas afectas. Por todo ello, es necesario que clínicos y cirujanos mejoren su conocimiento neural en un intento de aumentar la seguridad quirúrgica y prevenir lesiones del plexo lumbar.

\section{CONCLUSIONES}

1. El músculo psoas es una referencia quirúrgica básica en el abordaje retroperitoneal. Debe evitarse su borde más superior y lateral.

2. Los nervios GF (único o como dos ramas) y FCL pueden discurrir entre sus fascículos y deben ser localizados antes de actuar sobre el MP.

3. La gran variabilidad de formación en las raíces de los nervios terminales del plexo lumbar debe tenerse en cuenta al planear cualquier sección basada solo en el mapeo clínico.

4. El bloqueo del compartimento del psoas es una posibilidad de anestesia regional de gran espectro neural.

\section{BIBLIOGRAFÍA}

1. Newell RLM. Pelvic girdle and lower limb. In: Standing S (editor). Gray's Anatomy (39th Ed.). Philadelphia: Elsevier; 2005. pp: 1454-5.

2. Testut L, Latarjet A. Compendio de anatomía descriptiva (18. ${ }^{a}$ edición). Madrid: Salvat; 1920.

3. Lu S, Chang S, Zhang Y, Ding Z, Xu XM, Xu Y. Clinical anatomy and $3 \mathrm{D}$ virtual reconstruction of the lumbar plexus with respect to lumbar surgery. BMC Musculoskeletal Disorders. 2011;12:76.

4. Wang J, Zhou Y, Zhang ZF, Li CQ, Zheng WJ, Liu J. Minimally invasive or open transforaminal lumbar interbody fusion as revision surgery for patients previously treated by open discectomy and decompression of the lumbar spine. Eur Spine J. 2011;20:623-8.

5. Waschke A, Hartmann C, Walter J, Dunisch P, Wahnschaff F, Kalff $\mathrm{R}$, et al. Denervation and atrophy of paraspinal muscles after open lumbar interbody fusion is associated with clinical outcome-electromyographic and CT-volumetric investigation of 30 patients. Acta Neurochir (Wien). 2014;156:235-44.

6. Skovrlj B, Belton P, Zarzour H, Qureshi SA. Perioperative outcomes in minimally invasive lumbar spine surgery: A systematic review. World J Orthop. 2015;6:996-1005.

7. Houten JK, Alexandre LC, Nasser R, Wollowick AL. Nerve injury during the transpsoas approach for lumbar fusion. J Neurosurg Spine. 2011;15:280-4.

8. Cahill KS, Martínez JL, Wang MY, Vanni S, Levi AD. Motor nerve injuries following the minimally invasive lateral transpsoas approach. J Neurosurg Spine. 2012;17:227-31.

9. Dakwar E, Le TV, Baaj AA, Le AX, Smith WD, Akbarnia BA, et al. Abdominal wall paresis as a complication of minimally invasive lateral transpsoas interbody fusion. Neurosurg Focus. 2011;31:E18.

10. Benglis DM, Vanni S, Levi AD. An anatomical study of the lumbosacral plexus as related to the minimally invasive transpsoas approach to the lumbar spine. J Neurosurg Spine. 2009;10:139-44.

11. Ahmadian A, Deukmedjian AR, Abel N, Dakwar E, Uribe JS. Analysis of lumbar plexopathies and nerve injury after lateral retroperitoneal transpsoas approach: Diagnostic standardization. J Neurosurg Spine. 2013;18:289-97.

12. Rodgers WB, Gerber EJ, Patterson J. Intraoperative and early postoperative complications in extreme lateral interbody fusion: An analysis of 600 cases. Spine. 2011;36:26-32.

13. Capdevila X, Macaire P, Dadure C, Choquet O, Biboulet P, Ryckwaert Y, et al. Continuous psoas compartment block for postoperative analgesia after total hip arthroplasty: New landmarks, technical guidelines, and clinical evaluation. Anesth Analg. 2002;94:1606-13.

14. Mannion S, O'Callaghan S, Walsh M, Murphy DB, Shorten GD. In with the new, out with the old? Comparison of two approaches for psoas compartment block. Anesth Analg. 2005;101:259-64.

15. Aveline C, Bonnet F. Delayed retroperitoneal haematoma after failed lumbar plexus block. Br J Anaesth. 2004;93:589-91.

16. Tubbs RS, Salter EG, Wellons JC, Blount JP, Oakes WJ. Anatomical landmarks for the lumbar plexus on the posterior abdominal wall. Neurosurg Spine. 2005;2:335-8.

17. Banagan K, Gelb D, Poelstra K, Ludwig S. Anatomic mapping of lumbar nerve roots during a direct lateral transpsoas approach to the spine: A cadaveric study. Spine. 2011;36:E687-91.

18. Uribe JS, Arredondo N, Dakwar E, Vale FL. Defining the safe working zones using the minimally invasive lateral retroperitoneal transpsoas approach: An anatomical study. J Neurosurg Spine. 2010;13:260-6.

19. Guerin P, Obeid I, Gille O, Bourghli A, Luc S, Pointillart V, et al. Safe working zones using the minimally invasive lateral retroperitoneal transpsoas approach: A morphometric study. Surg Radiol Anat. 2011;33:665-71. 
20. Davis TT, Hynes RA, Fung DA, Spann SW, MacMillan M, Kwon $\mathrm{B}$, et al. Retroperitoneal oblique corridor to the L2-S1 intervertebral discs in the lateral position: An anatomic study. J Neurosurg Spine. 2014;21:785-93.

21. Moro T, Kikuchi S, Konno S, Yaginuma H. An anatomic study of the lumbar plexus with respect to retroperitoneal endoscopic surgery. Spine. 2003;28:423; discussion 427-8.

22. Moreno-Egea A. Surgical management of postoperative chronic inguinodynia by laparoscopic transabdominal preperitoneal approach. Surg Endosc. 2016;30(12):5222-7.

23. Moreno-Egea A. Neurectomía laparoscópica transabdominal preperitoneal como tratamiento de la inguinodinia. Experiencia personal y detalles de la técnica quirúrgica. Rev Hispanoam Hernia. 2018;6(2):69-74

24. Aida S, Takahashi H, Shimoji K. Renal subcapsular hematoma after lumbar plexus block. Anesthesiology 1996;84:452-5.

25. Mannion S, Barrett J, Kelly D, Murphy DB, Shorten GD. A description of the spread of injectate after psoas compartment block using magnetic resonance imaging. Reg Anesth Pain Med. 2005;30:567-71.

26. Heller AR, Fuchs A, Rossel T, Vicent O, Wiessner D, Funk RH, et al. Precision of traditional approaches for lumbar plexus block: Impact and management of interindividual anatomic variability. Anesthesiology. 2009;111:525-32.

27. Kaloul I, Guay J, Cote C, Fallaha M. The posterior lumbar plexus (psoas compartment) block and the three-in-one femoral nerve block provide similar postoperative analgesia after total knee replacement. Can J Anaesth. 2004;51:45-51.

28. Biboulet P, Morau D, Aubas P, Bringuier-Branchereau S, Capdevila $\mathrm{X}$. Postoperative analgesia after total-hip arthroplasty: Comparison of intravenous patient-controlled analgesia with morphine and single injection of femoral nerve or psoas compartment block. A prospective, randomized, double-blind study. Reg Anesth Pain Med. 2004;29:102-9.

29. Stevens RD, Van Gessel E, Flory N, Fournier R, Gamulin Z. Lumbar plexus block reduces pain and blood loss associated with total hip arthroplasty. Anesthesiology 2000;93:115-21.

30. Green C, Flannery O, Crotty J, Felle P, Harmon D, Masterson E. A Cadaveric study of injectate spread in the psoas compartment with a direct iliopsoas injection suggested for use during surgery. Clin Anat. 2011;24:763-7.

31. Van Campenhout A, Hubens G, Fagard K, Molenaers G. Localization of motor nerve branches of the human psoas muscle. Muscle Nerve. 2010;42:202-7.

32. Mahan MA, Sanders LE, Guan J, Dailey AT, Taylor W, Morton DA. Anatomy of psoas muscle innervation: cadaveric study. Clin Anat. 2017;30:479-86

33. Kirchmair L, Lirk P, Colvin J, Mitterschiffthaler G, Moriggl B. Lumbar plexus and psoas major muscle: Not always as expected. Reg Anesth Pain Med. 2008;33:109-14.

34. Sim IW, Webb T. Anatomy and anaesthesia of the lumbar somatic plexus. Anaesth Intensive Care. 2004;32:178-87. 